\title{
Gestaltung als Lebensbehauptung
}

\author{
Being Creative to Stay Alive
}

\author{
Autor \\ Reinhard Spieler \\ Institut \\ Sprengel Museum Hannover
}

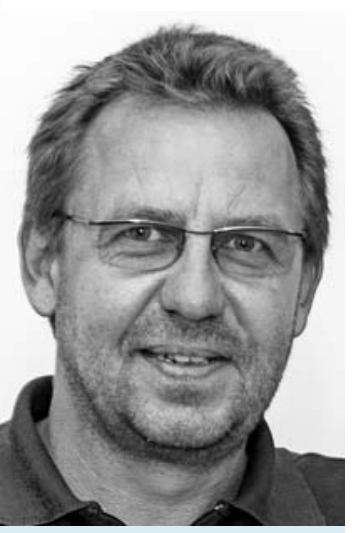

Reinhard Spieler (Quelle: Herling/Gwose/Werner, Sprengel Museum Hannover)

\section{Bibliografie}

DOI http://dx.doi.org/ 10.1055/s-0041-108226

Psychiat Prax 2016; 43: 8-9

(c) Georg Thieme Verlag KG

Stuttgart · New York

ISSN 0303-4259

Korrespondenzadresse Dr. Reinhard Spieler, Direktor Sprengel Museum Hannover Kurt-Schwitters-Platz 30169 Hannover reinhard.spieler@hannoverstadt.de

\section{Gestaltung als Lebensbehauptung}

Was erwarten wir von der Kunst? Zunächst einmal, dass sie Ausdrucksform für Gefühle und Gedanken ist, die uns als Mensch bewegen. Je authentischer und unmittelbarer dieser Ausdruck sich formuliert, je mehr die Künstlerin oder der Künstler von sich selbst zu geben vermag, umso eindrucksvoller und einprägsamer wirkt das Werk auf den Betrachter. Um überhaupt künstlerisch tätig zu werden, bedarf es eines Mitteilungsbedürfnisses; künstlerisches Gestalten wird dabei zu einem Mittel, sich selbst in der Welt zurechtzufinden, den eigenen Gefühlen und Wahrnehmungen eine Gestalt zu geben, die man nicht unbedingt verbal formulieren kann und die oft auch nicht als geistiges Konzept vorgedacht ist. Die gestaltete Form jedoch ermöglicht eine verbale und systematische Annäherung an die im Werk enthaltenen Themenkomplexe.

Die Schwierigkeit besteht darin, eine Balance zu finden aus Professionalität und erlernter Gestaltgebung einerseits und einer Unmittelbarkeit des Ausdrucks, die eben jene Professionalität hinter sich lässt. Im Laufe der Geschichte stand jahrhundertelang der Kanon von erlernter Formgebung an oberster Stelle. Erst im 20. Jahrhundert gerieten auch andere künstlerische Konzepte als ernst zu nehmende künstlerische Ausdrucksmöglichkeiten in den Blick. Der Surrealismus war die erste Bewegung in der Kunstgeschichte, die systematisch die Unmittelbarkeit des Ausdrucks suchte. In der écriture automatique galt es, alle Kontrolle des Intellekts auszuschalten. Der Idealzustand, den die Surrealisten für künstlerische Äußerungen suchten, war die Hysterie: ein Zustand fiebergleicher, erhöhter Sensibilität, in der man sich außerhalb des Kontrollmodus befindet, geleitet allein von Affekten, die aus dem Inneren an die Oberfläche drängen und einen zu unmittelbaren Aktionen und Handlungen drängen.

Im Zuge dieser Forderung gerieten auch die Ausdrucksartefakte von psychisch oder geistig erkrankten Menschen in den Fokus. Mit der Sammlung Prinzhorn nahm eine der weltweit wichtigsten Sammlungen aus diesem Bereich in den
1920er-Jahren Gestalt an - in genau der Zeit, in der auch der Surrealismus sich artikulierte. Allein die Terminologie zeigt, dass diese Ausdrucksformen nach wie vor ein Randdasein in der Kunst führen - bis heute ist von Outsider-Kunst die Rede. Gleichwohl gibt es immer wieder starke Befürworte und Verfechter dieser Kunstwerke. Harald Szeemann hatte die Sammlung Prinzhorn, die nach dem Zweiten Weltkrieg zunächst ein Schattendasein geführt hatte und in Vergessenheit geraten war, wiederentdeckt und in der Kunsthalle Bern ausgestellt. Zuletzt verhalf Massimiliano Gioni als Kurator der 50. Biennale von Venedig 2013 der Outsider-Art zu einem weltweit beachteten, höchst prominenten Auftritt auf der wohl wichtigsten Ausstellung für zeitgenössische Kunst. Die für die Titelseiten der Psychiatrischen Praxis ausgewählten Werke befinden sich also in bester Gesellschaft! [1]

\section{Einsamkeit - Angst - Schmerz}

$\nabla$

Das Spektrum der eingesandten und ausgewählten Arbeiten ist ebenso breit wie in ihrer Ausdruckskraft beeindruckend. Viele verarbeiten ganz offensichtlich tiefe Traumata - Schmerz, Angst und Isolation. Eindrucksvoll ist dabei vor allem, dass es nicht nur Erlebnisse sind, die pathologische Befindlichkeiten Einzelner betreffen, sondern Erfahrungen spiegeln, die jeder Mensch selbst einmal gemacht hat und daher unmittelbar nachvollziehen kann. Einsamkeit spielt z.B. eine zentrale Rolle in vielen Arbeiten, etwa von Menschen, die auf einer überdimensionalen Treppenanlage aneinander vorbeigehen - der eine nach oben, der andere nach unten, ohne Kommunikation, ohne bewusste Begegnung: die Treppe als Wüste der Einsamkeit. Andere Arbeiten skizzieren eine ähnliche Erfahrung als einsamen Weg nach Nirgendwo oder als Schweigen.

Ein Hauptthema vieler Arbeiten ist die Erfahrung von Angst. Sie äußert sich im Bild eines Strudels, der einen in die Tiefe zu ziehen droht (,Halt“), oder als Nachtmahr in Form eines Phantasievogels in grünblauer Nachtlandschaft, in der alles 
gespenstisch und bedrohlich wirkt. Eine andere Arbeit kombiniert Bild und Text, benennt die Angst vor Fremdem oder Fremden, vor Depression und Krankheit. In Suche nach Schutz manifestiert sich das Angstgefühl in einem Bild aus der Natur - ein Tier mit übergroßen Augen und Ohren, mithin ganz auf Sendung und Wahrnehmung eingerichtet, erfährt von einem Baum nicht nur Schutz und Geborgenheit, sondern scheint mit diesem Baum regelrecht zu verschmelzen, zu einer organischen Einheit zusammengewachsen. Dahinter ist es Hell formuliert ebenso die Angst vor dem Tod wie vielleicht auch die Zuversicht auf das, was sich nach dem (Ab)Leben offenbaren mag. Im Vordergrund dominieren dunkle Farben und kristallartige Strukturen, die einerseits skelettartige Stabilität bieten, andererseits aber auch wie ein Korsett Beengung und Unbeweglichkeit zum Ausdruck bringen. Im Zentrum hingegen breitet sich das pure Licht aus, das ohne jede Struktur und Binnenzeichnung auskommt und Raum für jede Form von Imagination bietet; es ist im wahrsten Sinne des Wortes ein Leerraum - bedrohlich und verheißungsvoll zugleich: ein eindrucksvolles Bild für ein Jenseits unseres Lebensbegriffes.

Die Angst vor einem Schlaganfall kommt in einem Gesicht zum Ausdruck, das von Bäumen und Wurzeln überlagert scheint, die vom Gesicht Besitz ergriffen haben, es übermächtig dominieren. Was auf den ersten Blick kraftvoll, in Pop-Art-Stil und Farben fast lebensbejahend wirkt, erweist sich beim genaueren Hinsehen als (Über)Lebenskampf mit einem aufgerissenen, noch wahrnehmenden Auge und einem anderen Auge, das schon gelähmt und abgestorben scheint. Trotz aller Bedrohung, die sich in dem Gesicht spiegelt, konstatiert das Bild dennoch eine Einheit von Mensch und Natur, wird die Bedrohung als ein Teil der Natur dargestellt - am Ende eine berührend versöhnliche Sicht auf diesen Zustand von existenzieller Angst.

Ein anderes Werk führt nicht minder eindrucksvoll den Gegensatz von Aufbau und Zerstörung, so der Titel, vor. Ein Baum aus lauter Gesichtern, der im Stamm ein blütentreibendes Herz trägt, wird bedroht von einem Räderwerk der Zerstörung. Ein menschlicher Körper, bestehend aus Zahnrädern, die mit der mechanischen Präzision eines Uhrwerks ein mörderisches Sägewerkzeug antreiben, rückt dem Baum mit seinen scharfen Zacken zu Leibe und droht abzuschneiden, was der Baum an Leben und Blütenpracht hervorbringt. Ebenso zerstörerisch und alles verschlingend zeigt sich die Angst vor dem Teufel Alkohol, der mit gieriger Zunge und stierem Blick nur ein Ziel zu haben scheint, nämlich alles in den Strudel der Sucht und der Gier hineinzuziehen. Als Beelzebub zeigt er die hässliche und bedrohliche Fratze von Angst, Gewalt und (Selbst-)Zerstörung.

Schmerz ist ein weiteres zentrales Thema vieler Arbeiten. Verzweifelte Gesten wie in Weinender Mensch, noch eindrucksvoller vielleicht sogar in der Zeichnung Fixierung führen extremes Leiden und Schmerz in einer eindrucksvoll verdichteten Form vor. Kleinteilige Zeichnungen mit spitzem Stift und scharfen Linien führen neuronale Vernetzungen, gewissermaßen ein flächendeckendes Netzwerk der Schmerzen vor; eine Spirale aus Blut und Tränen lässt das Drama der Ausweglosigkeit erahnen. Jeder hat sein Päckchen zu tragen, und diese Päckchen erweisen sich als schwere, fast untragbare Last: Belasteter Mensch führt uns das in der vordergründig harmlosen Form einer Kinderzeichnung vor und doch in einer berückenden Intensität, für die man sich kaum ein stärkeres Bild vorstellen könnte. Tragen und Getragen-Sein offenbart uns, dass das Tragen einer schweren Last durchaus auch als positives Gegengewicht das Gefühl von Zusammengehörigkeit und Vertrauen beinhalten kann.

\section{Ausdrucksmittel \\ $\nabla$}

Während das Themenspektrum der eingereichten Arbeiten sich weitgehend in Problembereichen bewegt und Angst-, Schmerzund Isolationserlebnisse zu verarbeiten sucht, weisen die Ausdrucksmittel eine große Bandbreite auf. Das beinhaltet zum einen die Rückgriffe auf bekannte Positionen der Kunstgeschichte. Insbesondere Künstler, die eine Sprache zeichenhafter, symbolischer Verdichtung entwickelt haben, scheinen die Schöpfer der Titelbilder hier besonders beeinflusst und inspiriert zu haben. Paul Klee wäre hier an erster Stelle zu nennen, ebenso könnte man auch an Miró oder in der jüngeren Kunst an Keith Haring oder A. R. Penck denken. Auf ganz andere Art und Weise, mit fotografischen Mitteln, erkennt ein Künstler in Kanaldeckeln solche symbolisch verdichteten Figurationen.

Manchmal geht diese zeichenhafte Abstrahierung einher mit einer betonten Materialdichte, mit pastos aufgetragener Farbe, die der Art Brut, etwa einem Jean Dubuffet nahesteht. Die Materialhaftigkeit bewirkt einen sehr unmittelbaren Ausdruck, der keinen Umweg über ausgewogene oder ausgetüftelte Komposition und Linienführung benötigt.

Grundsätzlich lassen sich bei den Ausdrucksmitteln zwei große Grundrichtungen beobachten. Die einen setzen sehr stark auf die Wirkung der Linie. Das beinhaltet die symbolhaften Strichfiguren in der Nachfolge von Klee, Miró oder Haring, aber es betrifft auch vor allem viele, die mit dem Stift arbeiten. Die scharfe, präzise Linie transportiert oft eine Wirkung von Verletzung oder Verletzbarkeit, geht meist mit einem sehr expressiven Duktus einher.

Die andere Fraktion setzt mehr auf die Farbe und lässt an kunstgeschichtliche Vorbilder wie etwa Emil Nolde, aber zum Teil auch der Farbfeldmalerei denken. Die unmittelbare emotionale Wirkung der Farbe steht hier im Vordergrund. Selbstverständlich gibt es auch die Verbindung von Farbe und Linie - zum Teil im Stile der Pop-Art, aber oft begegnen wir auch Künstlerinnen und Künstlern, die stilistisch der naiven Kunst nahestehen. Die Verbindung zwischen naiver Kunst und Kinderzeichnung ist eng und wurde sowohl von der Art Brut als auch später von Künstlergruppen wie SPUR, Cobra und anderen verfolgt, die auch dem oft direkten, unkalkulierten Ausdruckspotenzial von Menschen mit psychischen Erkrankungen nahestehen.

In der thematischen Fokussierung sind diese Arbeiten beeindruckende Zeugnisse meist tiefgreifender, traumatischer Erfahrungen, die alle Menschen angehen und zu berühren vermögen. Sie beeindrucken aber ebenso in ihren künstlerischen Mitteln, die zum Teil an große Vorbilder in der Kunstgeschichte anknüpfen, die aber vor allem adäquate Ausdrucksmittel für ihre inhaltlichen Anliegen finden. Obwohl es meist um negative Erfahrungen geht, kommt in allen Arbeiten letztlich doch ein Lebenswille, ein Verarbeitungs- und Ausdruckswille zum Vorschein, in dem sich eine positive Haltung zum Leben manifestiert, so wie es Thomas Mann einst in einem Brief an den Philosophen Karl Kerenyi formulierte: „Ein Werk, und sei es eines der Verzweiflung, kann immer nur den Optimismus, den Glauben ans Leben zur letzten Substanz haben“ " [2].

\footnotetext{
Literatur

1 Becker T. „Alle machen mit“ - Titelbildwettbewerb geht in die zweite Runde. Psychiat Prax 2014; 41: 410-411

2 Kerenyi K, Hrsg. Thomas Mann - Karl Kerenyi. Gespräch in Briefen.
} Zürich: Rhein Verlag; 1960: 146 